\title{
Compromising Science by Ignorant Instrument Calibration-Need to Revisit Half a Century of Published XPS Data
}

Grzegorz Greczynski and Lars Hultman

The self-archived postprint version of this journal article is available at Linköping University Institutional Repository (DiVA):

http://urn.kb.se/resolve?urn=urn:nbn:se:liu:diva-164613

N.B.: When citing this work, cite the original publication.

Greczynski, G., Hultman, L., (2020), Compromising Science by Ignorant Instrument Calibration-Need to Revisit Half a Century of Published XPS Data, Angewandte Chemie International Edition.

https://doi.org/10.1002/anie.201916000

Original publication available at:

https://doi.org/10.1002/anie.201916000

Copyright: Wiley (12 months)

http://eu.wiley.com/WileyCDA/ 


\title{
Compromising science by ignorant instrument calibration - need to revisit half a century of published XPS data
}

\author{
G. Greczynski* and L. Hultman \\ Thin Film Physics Division, Department of Physics, Chemistry, and Biology (IFM), \\ Linköping University, \\ SE-581 83 Linköping, Sweden
}

X-ray photoelectron spectroscopy (XPS) is an indispensable technique in modern materials science for the determination of chemical bonding as evidenced by more than 10000 XPS papers published annually. A literature survey reveals that in the vast majority of cases an incorrect referencing of the binding energy scale is used, neglecting warnings that have been formulated from the early days of the technique. Consequences for the data reliability are disastrous and decades of XPS work require revisiting. The purpose of this Viewpoint is to highlight the existing problems, review the criticism and suggest ways forward.

XPS is based on the photoelectric effect, i.e., emission of electrons from surfaces exposed to light of sufficiently high energy. ${ }^{1}$ As an electrons inelastic mean free paths for energies involved are of the order of few $\mathrm{nm}$, the technique is characterized by high surface sensitivity. By measuring kinetic energy of electrons that leave the surface without inelastic collisions one can obtain a precise estimate of core level binding energy (BE) through the use of the Einstein relation..$^{2}$ Importantly, since the charge density on the atom affects binding energy of core level electrons any changes in the chemical environment are directly manifested in the XPS spectrum as peak shifts (chemical shifts). ${ }^{3,4}$ This allows for determination of bonding structure and the changes thereof as a function of processing parameters or surface treatments, by comparison of measured $\mathrm{BE}$ values to literature data bases. ${ }^{5}$ The possibility to obtain surface chemistry, 
composition for all sorts of specimens (thin films, solids, powders, gels, liquids, gases) together with apparent simplicity makes XPS rather unique and accounts for tremendous increase in the popularity of this technique in materials science (see Fig. 1).

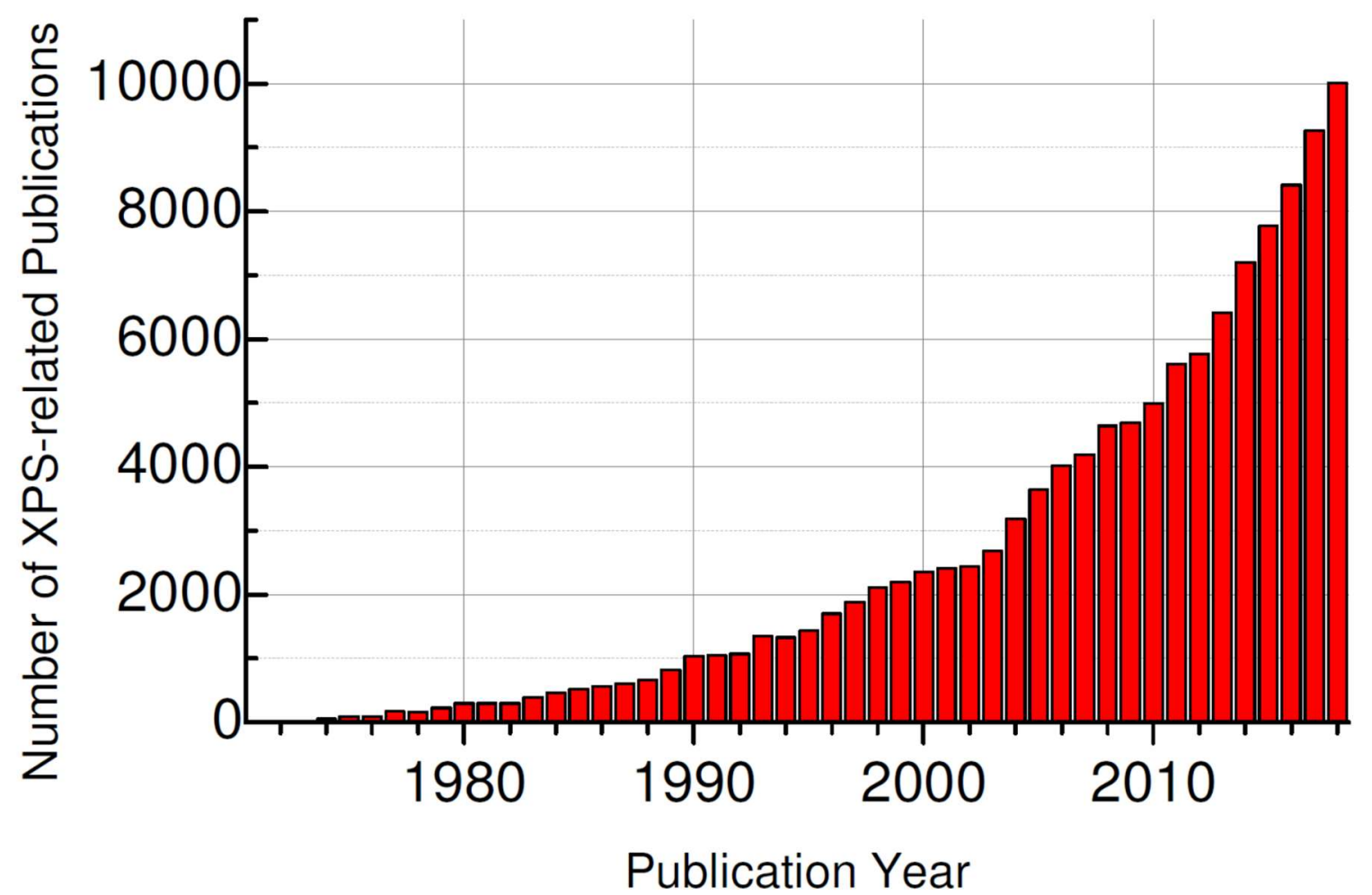

Fig. 1 Number of publications per year where XPS was used based on a Scopus data base search performed in November 2019 for the term "XPS".

\section{Hidden variables}

The first necessary condition to make an accurate determination of the BEs from the XPS spectrum is that the energy scale of the spectrometer is correctly calibrated. ${ }^{6}$ This standardized procedure involves measurements of specific core level signals from metal samples under well-defined conditions and proper adjustment of the spectrometer BE scale so that BE's of reference peaks agree with the recommended values. If the calibration process is correctly performed, the Fermi edge of metallic samples that remain in electrical contact to the spectrometer coincide with the "0 $\mathrm{eV}$ " of the BE scale (see Fig. 2), as both bodies share a common Fermi level (FL), which becomes a natural reference level. The FL alignment is easily 
verified in the case of metallic samples characterized by given density-of-states (DOS) at the FL.

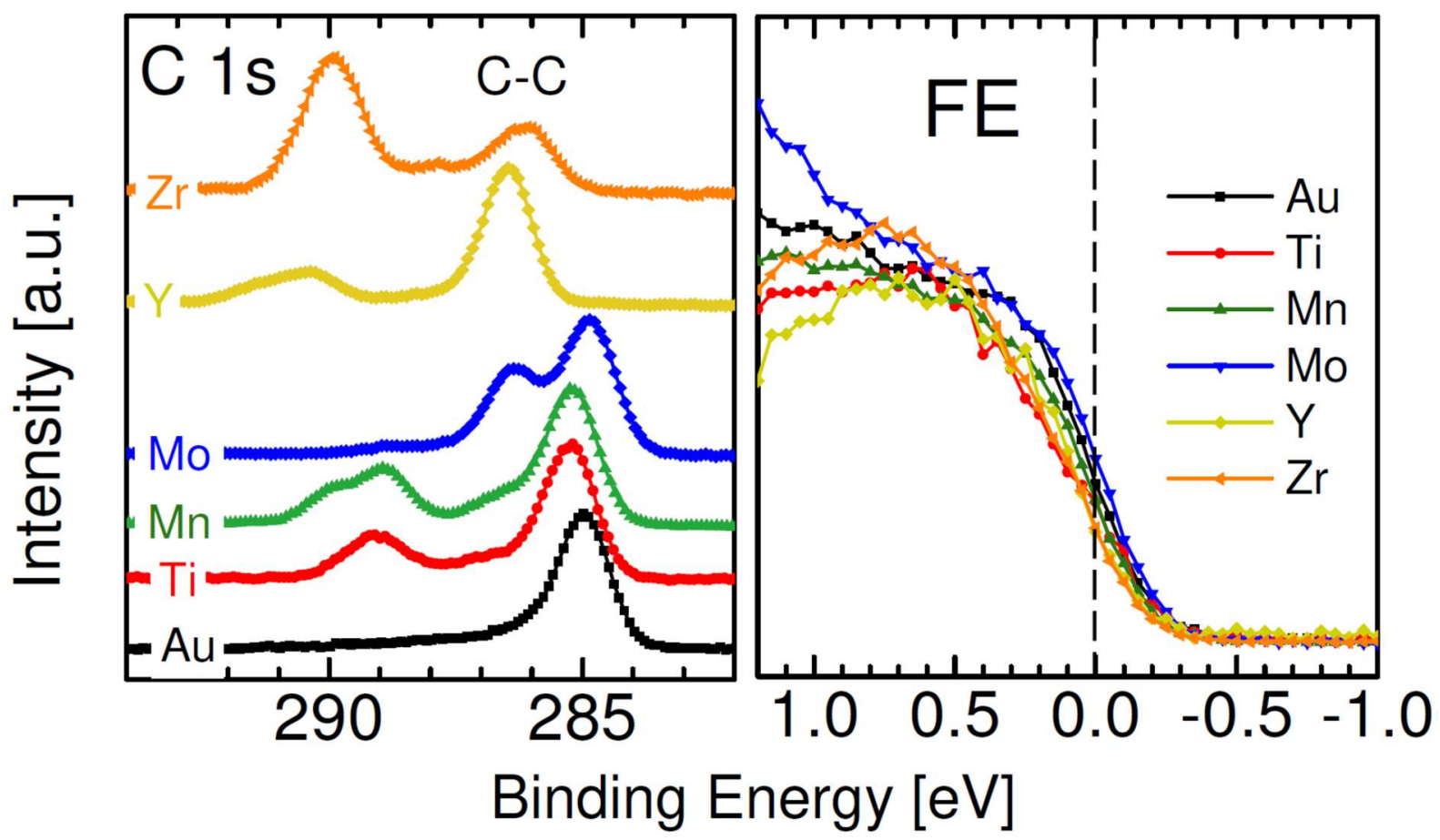

Fig. 2 (a) C 1s spectra recorded from $\mathrm{Au}, \mathrm{Ti}, \mathrm{Mn}, \mathrm{Mo}, \mathrm{Y}$, and $\mathrm{Zr}$ surfaces exposed to laboratory air for the time period of one day, and (b) corresponding XPS valence band spectra in the vicinity of Fermi level (FL). In all cases FL cut-off coincides with " 0 " eV on the BE scale, indicative of that FL is aligned between sample and the spectrometer. [Adopted from Ref. 20]

However, the situation changes dramatically is the case of poor conductors. The FL alignment between sample and the spectrometer can only take place if there is a sufficient charge density in the sample so that once brought in contact with the instrument, charge can flow across the interface until the equilibrium state is reached. If, for any reason (like low electrical conductivity or poor electrical contact) this condition is not fulfilled, the FL of the sample is decoupled from that of the spectrometer leading to incorrect BE values. The problem is that with exception for metals, other types of samples like semiconductors and/or insulators lack DOS at the FL, which prevents direct verification whether FL are aligned.

Another complication arising for poorly conducting specimens is that the surface potential during the measurement is not known a priori. Once X-rays are hitting the sample and photoemission process sets in, the loss of electrons from the surface has to be compensated at 
a high-enough rate to preserve charge neutrality. If that is not the case the surface acquires a positive potential, which decreases the kinetic energy of escaping photoelectrons, and results in the shift of all core-level peaks towards higher BE (sample charging). The latter can range from just tenths of an $\mathrm{eV}$, in which case it may go unnoticed, to several hundred $\mathrm{eV}$ for insulators, where essentially no photoelectrons leave the surface. Obviously, under such circumstances, the natural reference level is lost.

The lack of possibility to verify FL alignment and the unknown charging state during measurement are fundamental problems of the technique, which accounts for the fact that the reported binding energies for primary core-levels of constituting elements in many technologically-relevant materials exhibit an unacceptably large spread, often larger than the magnitude of related chemical shifts in the studied material. For example, the BE spread in the NIST XPS data base is in the range $0.7 \leq \Delta \mathrm{BE} \leq 3.1 \mathrm{eV}$ for nitrides, $0.8 \leq \Delta \mathrm{BE} \leq 3.6 \mathrm{eV}$ for sulfides, $0.5 \leq \Delta \mathrm{BE} \leq 8.5 \mathrm{eV}$ for fluorides, and $0.6 \leq \Delta \mathrm{BE} \leq 4.1 \mathrm{eV}$ for oxides. ${ }^{7}$ Even for metallic specimens $\Delta \mathrm{BE} \sim 1 \mathrm{eV}$, which corresponds to a typical chemical shift. A direct consequence of such large variations in reported core-level BEs is an incorrect bonding assignment, an arbitrary spectral interpretation, and, in the end, contradictory and often unreliable results. The problem grows with the advancements in the materials science, which often lead to that more complex materials are analyzed, with multiple chemical states of the same element, which in turn imposes very high requirements for the measurement accuracy. Thus, the risk of data misinterpretation is an increasing function of system complexity, which presents a formidable stumbling block in the practice of XPS spectral deconvolution.

\section{The C 1s method}

So, how is the BE referenced for samples other than metals? As a matter of fact, what may appear hard to believe, the accuracy of chemical state determination depends in the 
majority of cases on a sub-nm contamination layer that accumulates essentially on all airexposed surfaces, the so-called adventitious carbon (AdC). The method is as old as the XPS itself and has been suggested by the Nobel Price Laurate K. Siegbahn in his famous book on electron spectroscopy that appeared in 1967 and which marks the start of chemical analysis by electron spectroscopy. In there, one can read that: "[...] we have found the carbon 1s line from the pump oil ideally suited for use as a calibration line. In fact, there is usually no difficulty in distinguishing this line from the rest of the spectrum since its relative intensity increases with time." ${ }^{8}$ The method is, in other words, fairly simple - the C 1s line of AdC is deliberately set at $285.0 \mathrm{eV}$ and all other signals are shifted accordingly. The ad hoc assignment of AdC origin to diffusion pump oil, not backed up with any attempt of chemical identification, and neglecting other obvious sources like atmosphere exposure and sample handling, is only a minor issue if considered in the context of consequences brought about by the claim of constant binding energy of the C 1s peak - a myth that dominated XPS analyses for more than half the century.

\section{Criticism}

It did not take long for the first explicit criticism to the C 1s method to appear. In 1970, three years after the publication of the Siegbahns book, Nordling et al. ${ }^{9}$ suggested that the binding energy of the $\mathrm{C} 1 \mathrm{~s}$ line from $\mathrm{AdC}$ can be affected by the polarization of the thin layer and proposed mixing specimen with a reference such as graphite to enhance reliability. One year later, Hnatowich et al. got right to the point that the use of substances of unknown and potentially varying composition for energy referencing is a highly questionable procedure, especially because the electrical equilibrium between adsorbed AdC layer and specimen was not demonstrated. ${ }^{10}$ This was backed up by Dianis et al. ${ }^{11}$ who concluded that the $\mathrm{C} 1 \mathrm{~s}$ BE for $\mathrm{AdC}$ is highly uncertain and cannot be used for reference purposes. They also wrote that $\mathrm{C}$ can react on catalytically active surfaces which adds to confusion as: "... the interaction of the 
vapor with the active surface might shift the binding energy of the carbon. Therefore, one cannot be certain that one is looking at the same type of carbon every time."

As the result of this criticism wave from early 70's in the review of XPS calibration methods, Johansson et al issued a rather careful warning "Although different experiments have given the same $C 1$ s binding energy, there have been objections to this procedure, since the contamination has not been fully identified". ${ }^{12}$ This was likely the last effort to defend the claim of a constant $\mathrm{BE}$ of the $\mathrm{C} 1 \mathrm{~s}$ line. From this point on all papers that take upon the subject refer to the BE range which implies breaking a certain psychological barrier by admitting that $\mathrm{C} 1 \mathrm{~s}$ 's peak position may indeed vary - nothing one could expect for the proper reference.

Objections to the method did not stop at that. In 1976 the claim of constant BE of the C 1s peak was questioned by Kinoshita et al. ${ }^{13}$ who studied adventitious carbon adsorbed on an in situ deposited Au surface and concluded that "[...] the energy of the C 1s peak of contamination carbon adsorbed on metal surface can vary depending on the amount of adsorption, consequently its use as the reference of energy calibration seems to be dubious in some cases, especially for metallic samples." Cautioning voices did not cease even into the next decade. In the literature review with a rather rhetoric title "Adventitious Carbon-The Panacea for Energy Referencing?" published by Swift in $1982,{ }^{14}$ concerns were formulated in a more explicit way: "although the use of $C 1 s$ electrons from adventitious carbon layers is often a convenient method of energy referencing, interpretation of binding energy data obtained should be treated with caution". Unfortunately, few took this warning seriously and occasionally reported BE reference problems ${ }^{15,16,17}$ literally drowned in the sea of XPS literature based on the C 1s referencing.

\section{Status Quo}

The survey of the XPS literature covering the last 20 years reveals that roughly $60 \%$ of all XPS-related papers explicitly admit using C 1s peak of AdC as the BE reference, despite all 
existing criticism. This does not mean that the remaining $40 \%$ comes from more careful labs, alarmingly, these papers simply lack information about any referencing method at all.

Furthermore, the detailed analysis of how the $\mathrm{C} 1 \mathrm{~s}$ referencing is actually performed reveals large discrepancies to an extent that prevents any meaningful inter-laboratory comparison of the obtained results. The problems can be divided in four categories: (1) the claimed type of contamination species used for calibration, (2) the way method is applied, (3) lack of understanding when the method is necessary, and (4) the BE assigned to the reference C $1 \mathrm{~s}$ peak.

Firstly, that there is no apparent consensus as to the nature of the carbon species that are used for calibration and terms that appear most often include "adventitious carbon", "graphitic carbon", "aliphatic carbon", or "hydrocarbons". These are, however, not confirmed by means of proper chemical analyses of surface species, but rather ironic assumptions based on earlier claims. Equally disturbing are completely unjustified adjectives like "uncharged AdC" or "neutral AdC" that serve as examples of wishful thinking. The C 1s spectra are reported only if they contain contributions from the primary material to be analyzed. Thus, in general, referencing is performed using the C 1s signal from an unknown compound.

Secondly, while referring to the C 1s method authors are claiming "calibration", "referencing" and "correcting" the BE scale, which are, obviously, not the same things. Far more controversial terms appear. For example, BE scale is "normalized to the $\mathrm{C} 1 \mathrm{~s}$ ", "set by fixing the C 1s component", "taken as a standard", "adjusted", or "set". Presumably, all authors mean the same procedure, nevertheless improper or inadequate description undermines the already ill-defined method.

Third issue is that in many cases $\mathrm{C} 1 \mathrm{~s}$ method with its all well-documented problems is used even though better alternatives exists. This is commonly the case for analyses performed on 
conducting samples, often with well-defined FL cut-off, which calls to be used as a BE reference. This clearly shows that XPS education has to be improved.

The fourth, and the most alarming issue, has to do with the large spread in the BE values assigned to the $\mathrm{C} 1 \mathrm{~s}$ peak, which is arbitrary set anywhere between 284.0 and $285.6 \mathrm{eV}$. This serious inconsistency contradicts the notion of a BE reference, which per definition should be based on one (unique) value. The arbitrary character of the method results in incorrect spectral interpretation, contradictory results, and generates a large spread in reported BE values for elements even present in the same chemical state. Owing to the huge volume of XPS literature produced each year (see Fig. 1), the consequences of such status quo are dramatic.

In view of the numerous and ineligible reports on problems, it is highly disturbing that the C 1s method has remained so popular. While it can be to some extent understood that the poor energy resolution in the early XPS days potentially prevented realization that the BE of AdC C 1s peak is not constant, it is beyond comprehension why this fact, once reported, did not disprove the applicability of this approach among peers and journal editors alike.

There are several factors that can be potentially responsible for this situation: (1) no good alternative to the $\mathrm{C}$ 1s method exists for poorly conducting samples, (2) AdC is present on all air-exposed surfaces so the method is fairly simple and, thus, compelling, and (3) last but not least, both ASTM and ISO recommend C 1s method for referencing, ${ }^{18,19}$ with only a brief disclaimer: "A significant disadvantage of this method lies in the uncertainty of the true nature of the carbon and the appropriate reference values which, as reported in the literature, have a wide range from $284.6 \mathrm{eV}$ to $285.2 \mathrm{eV}$ for the C 1 s electrons from hydrocarbon and graphitic carbon.[...]".

\section{Fighting the myths}

Recent systematic studies showed undoubtedly that the chemical nature of adsorbing AdC depends on the substrate, the type of environment it has been exposed to, and the exposure 
time. ${ }^{20,21} \mathrm{C}$ 1s spectra recorded from several metal samples exposed to air shown in Fig. 2 reveal large differences both in the type as well as in the number of $\mathrm{C}$ chemical states. Moreover, the $\mathrm{BE}$ of the $\mathrm{C} 1 \mathrm{~s}$ peak assigned to $\mathrm{C}-\mathrm{C}$ bonds exhibits large changes. A large study including carefully selected set of nearly one hundred thin-film samples spanning a wide range of material systems representing metals, nitrides, carbides, borides, oxides, and oxynitrides reveals that the $\mathrm{BE}$ of the $\mathrm{C}-\mathrm{C} / \mathrm{C}-\mathrm{H}$ peak of $\mathrm{AdC}$ depends on the substrate it accumulates on and may vary by as much as $2.66 \mathrm{eV}$ for the range of tested materials systems. ${ }^{22}$ Hence, the magnitude of $\mathrm{C} 1 \mathrm{~s}$ shifts caused by the substrate is larger than typical chemical shifts, which definitely prevents any meaningful bonding assignments. Setting the $\mathrm{C} 1 \mathrm{~s}$ peak at arbitrary chosen BE value from the range suggested by ISO standard, $284.6-285.2 \mathrm{eV}$, is not justified and may lead to unphysical results, like a non-zero density of states above the Fermi level. ${ }^{23}$

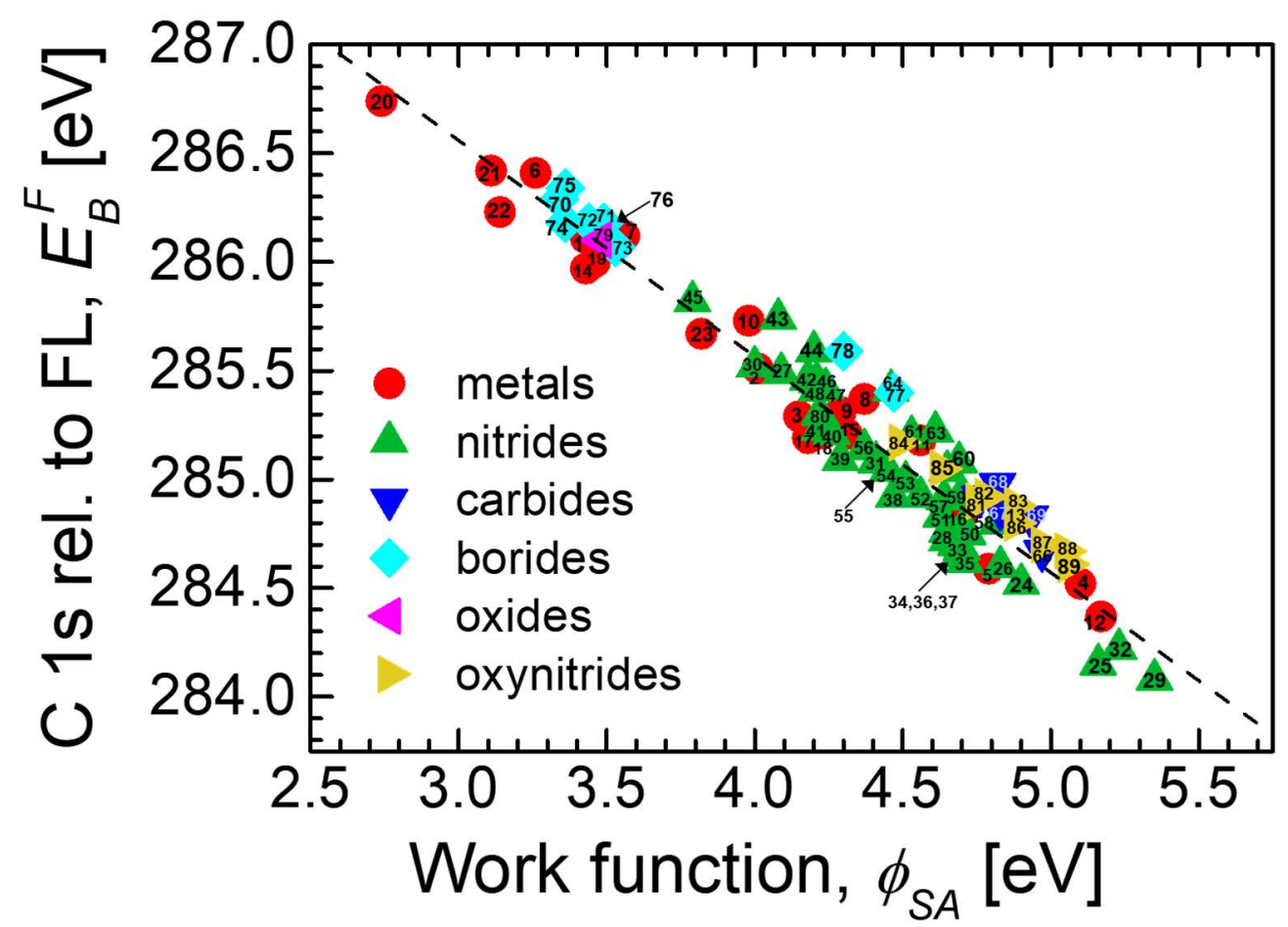

Fig. 3 Binding energy of the $\mathrm{C} 1 \mathrm{~s}$ peak of $\mathrm{AdC} E_{B}^{F}$ plotted vs. sample work function $\phi_{S A}$ for the carefully selected set of nearly one hundred predominantly thin film samples spanning wide range of material systems representing metals, nitrides, carbides, borides, oxides, and oxynitrides.[Adopted from Ref. 22]

But, why does the $\mathrm{C} 1 \mathrm{~s}$ peak shift so much from substrate to substrate? Interestingly, in the same set of experiments that revealed the magnitude of these shifts, the close correlation 
between the measured $\mathrm{BE}$ of the $\mathrm{C} 1 \mathrm{~s}$ peak from adventitious carbon $E_{B}^{F}$ and the sample work function $\phi_{S A}$, was demonstrated. ${ }^{22}$ As depicted in Fig. 3, within the measurement accuracy the $\operatorname{sum} E_{B}^{F}+\phi_{S A}$ is constant. Since the latter quantity represents the BE referenced to the vacuum level (VL), one can conclude that in the case of AdC layer adsorbed on the whole range of thin film samples the VL alignment, rather than commonly assumed FL alignment, takes place. This indicates that no charge transfer takes place between AdC layer and the surface it adsorbs on, which can be intuitively understood as AdC is not an inherent part of the analyzed sample and as such may not remain in a proper electrical contact with the substrate (and spectrometer). The bottom line is that the apparent $\mathrm{BE}$ of the $\mathrm{C} 1 \mathrm{~s}$ peak is steered by the sample work function, which is a very sensitive quantity, and as such cannot serve as a reliable reference for calibrating the BE scale.

\section{Outlook}

In view of all existing experimental evidence, the $C$ 1s peak of adventitious carbon can not serve as the BE reference in XPS. Unfortunately, no reliable alternative exists. No method is, however, better than an incorrect method. The historical perspective sketched above reveals a great deal of nonchalance which lead to that warning signals appearing from various laboratories were largely neglected in the "main stream" XPS literature.

The only justified use of the $\mathrm{C}$ 1s peak of $\mathrm{AdC}$ for $\mathrm{BE}$ referencing is that for layers accumulated on conducting substrates and that only provided that the simultaneous measurement of the sample work function can be performed. In such case the $\mathrm{C}-\mathrm{C} / \mathrm{C}-\mathrm{H}$ component can be set at $289.58-\phi_{S A} \mathrm{eV}$ and all other core-levels shifted accordingly. This procedure allows a user to maintain the concept of a single specific BE value associated with the $\mathrm{C}$ 1s peak of AdC, however, only for conducting samples, in which case natural reference to the FL can be performed. 
To change the status quo, better attention is required from peers and journal editors, who should challenge the provided description of the experimental XPS procedures in a submitted manuscript. This would also help to address other common issues like incorrect spectra fitting (ignoring proper background functions and line shapes), differential charging, or sputter damage, all of which are beyond the scope of this paper.

The charge referencing guidelines published by both ASTM and ISO, have to be revisited promptly with stronger warning, recognizing the external nature of AdC layers, which typically exhibit weak interaction to the underlying specimen and, in consequence, do not align to the Fermi level of the spectrometer.

To end on the positive note, experts seem to be well aware of $\mathrm{C} 1 \mathrm{~s}$ referencing issues; ${ }^{24,25}$ the challenge is to convey this attitude to new generations of XPS practitioners to stop the avalanche of XPS papers with incorrectly referenced spectra. The first step to solve the problem is to be aware of it. Once the issue is widely recognized perhaps alternative solutions to the $\mathrm{C}$ 1s method will be developed leading to the new consensus for standards. Potential candidates include noble metal decoration, ${ }^{10}$ implantation of noble gas atoms, ${ }^{26}$ or chemical state assessment based on the Auger parameter. ${ }^{27}$

\section{Acknowledgements}

The authors most gratefully acknowledge the financial support of the Knut and Alice Wallenberg Foundation Scholar Grant KAW2016.0358, the VINN Excellence Center Functional Nanoscale Materials (FunMat-2) Grant 2016-05156, the Swedish Research Council VR Grant 2018-03957, the Åforsk Foundation Grant 16-359, and Carl Tryggers Stiftelse contract CTS 17:166.

\section{References}




\footnotetext{
${ }^{1}$ Hertz H, Ann. Phys. U. Chem. (Wied. Ann) 1887;31:421

${ }^{2}$ Einstein A, On a Heuristic Point of View about the Creation and Conversion of Light. Ann. Phys. 1905;17: $132-148$.

${ }^{3}$ Sokolowski E, Nordling C, Siegbahn K. Chemical shift effect in inner electronic levels of Cu due to oxidation. Physical Review 1958;110:776.

${ }^{4}$ Fahlman A, Hamrin K, Hedman J, Nordberg R, Nordling C, Siegbahn K. Electron spectroscopy and chemical binding. Nature 1966;210:4-8.

${ }^{5}$ Hüfner S, "Photoelectron Spectroscopy: Principles and Applications", Springer-Verlag, 2003.

${ }^{6}$ ISO 15472:2010, "Surface chemical analysis -- X-ray photoelectron spectrometers -- Calibration of energy scales", (ISO, Geneva, 2010)

${ }^{7}$ NIST X-ray Photoelectron Spectroscopy Database, Version 4.1 (National Institute of Standards and Technology, Gaithersburg, 2012); http://srdata.nist.gov/xps/. Accessed: 2018-03-02

${ }^{8}$ Siegbahn K, Nordling C. ESCA, atomic, molecular and solid state structure studied by means of electron spectroscopy. Nov. Act. Uppsaliensis. 1967.

${ }^{9}$ Nordberg R, Brecht H, Albridge RG, Fahlman A, Van Wazer JR. Binding energy of the" 2p" electrons of silicon in various compounds. Inorganic Chemistry. 1970;9:2469-74.

${ }^{10}$ Hnatowich DJ, Hudis J, Perlman ML, Ragaini RC. Determination of charging effect in photoelectron spectroscopy of nonconducting solids. Journal of Applied Physics. 1971;42:4883-6.

${ }^{11}$ Dianis WP, Lester JE. External standards in x-ray photoelectron spectroscopy. Comparison of gold, carbon, and molybdenum trioxide. Analytical Chemistry. 1973;45:1416-20.

12 Johansson GG, Johansson J, Hedman A, Berndtsson M, Klasson R, Nilsson. Journal of Electron Spectroscopy and Related Phenomena 1973;2:295.

${ }^{13}$ Kinoshita S, Ohta T, Kuroda H. Comments on the Energy Calibration in X-Ray Photoelectron Spectroscopy. Bulletin of the Chemical Society of Japan. 1976;49:1149-50.

${ }^{14}$ Swift P. Surface and Interface Analysis. 1982;4:47-51.

${ }^{15}$ Werrett CR, Bhattacharya AK, Pyke DR. Applied Surface Science. 1996;103:403-7.

${ }^{16}$ Peplinski B, Unger WE, Grohmann I. Applied Surface Science. 1992;62:115-29.

${ }^{17}$ Gross T, Ramm M, Sonntag H, Unger W, Weijers HM, Adem EH. Surface and Interface Analysis. 1992;18:59-64.

${ }^{18}$ ASTM E1523-15, Standard Guide to Charge Control and Charge Referencing Techniques in X-Ray

Photoelectron Spectroscopy, ASTM International, West Conshohocken, PA, 2015, www.astm.org

${ }^{19}$ ISO 19318:2004 "Surface chemical analysis - Reporting of methods used for charge control and charge correction"

${ }^{20}$ Greczynski G, Hultman L. Prog. Mats. Sci. 107 (2020) 100591

${ }^{21}$ Crist BV. XPS in industry - Problems with binding energies in journals and binding energy databases. J. Electron Spectrosc. Relat. Phenom. 2019;231:75.

${ }^{22}$ Greczynski G, Hultman L. Applied Surface Science. 2018;451:99-103.

${ }^{23}$ Greczynski G, Hultman L. ChemPhysChem. 2017;18:1507-12.

${ }^{24}$ D. R. Baer et al, J. Vac. Sci. Technol. A 37 (2019) 031401

${ }^{25}$ C.J. Powell, J. El. Spec. Rel. Phenom. (2020), in press

${ }^{26}$ P.H. Citrin, D.R. Hamann, Phys Rev B 10 (1974) 4948

${ }^{27}$ C.D. Wagner, Faraday Discuss Chem Soc. 60 (1975) 291-300
} 